\title{
PERSPECTIVES
}

OPINION

\section{Challenges to cancer control by screening}

\author{
M ichael N. Pollak and William D. Foulkes
}

Population-based screening seems to be a common-sense strategy for controlling cancer, but recent reports have raised controversy concerning the benefits of common screening procedures. Intense efforts to develop and evaluate novel screening technologies are underway; however, effective use of any screening method must take into account any underlying biological considerations. What are these biological issues, and what challenges do clinicians face in screening for common cancers?

Screening populations for the presence of an asymptomatic illness will clearly be useful if the screening method is convenient and inexpensive, if false negatives and false positives are rare, and if treatment that was initiated at the early, screening-detected stage is more successful than treatment initiated later in thenatural history of theillness. Although it is true that the outcome of cancer treatment is almost al ways more favourable when treatment is initiated early, it is simplistic to extrapolate that, in general, population screening will providea practical method for cancer control. Indeed, recent reports have raised controversy concerning the benefits of common screening procedures, such as breast self-examination (see TIM ELINE), mammography and prostate-specific antigen (PSA) measurements ${ }^{1-3}$.

Important issues includelack of benefit of early detection of disease that cannot be effectively treated and, conversely, early detection of conditions that have a low or unknown probability of causing clinical diseaselater in life. Screening procedures must be evaluated not only from thepoint of view of individuals at risk, but also from the point of view of populations at risk - conclusions obtained from rigorous assessments of screening methods might not always correspond to expectations.

As long as our ability to treat advanced cancer remains inadequate, therewill beobvious pressureto implement effective screening strategies, and new technologies for detecting cancer - for example, spiral computed tomography (CT) imaging for lung cancer detection and proteomic serum assays for ovarian cancer detection - arebeing developed at present ${ }^{4,5}$. However, success of a screening method in detecting cancers before they are clinically apparent is not necessarily sufficient for a screening method to be useful.

\section{Biological issues affect screening} Often, research agendas that are designed to improvecancer screening methods emphasize technology development (for example, serum proteomics or high-definition digital mammography), and assign relatively little attention to the biological characteristics of cancers that influencethe efficacy of screening efforts. Yet, the task of developing and implementing effective screening-based methods for cancer control is challenging for biological reasons that deserve consideration.

Stepwise progression to malignancy. The fact that carcinogenesis rarely involves a single transforming step from normal cells to aggressive cancer cells complicates the issue of screening. A method that identifies only fully transformed neoplastic cells with metastatic potential might indeed result in earlier detection than would be the case if patients were assessed only by clinical examination. However, a significant proportion of such lesions might have al ready resulted in micrometastases when detected, thereby limiting the benefits of early surgical removal and the entire screening exercise (FIG. 1). Although many screen-detected cancers have good prognostic features ${ }^{6}$, the fact that a cancer is detected by screening does not guarantee curability.

Screening methods that attempt to avoid this hazard by detecting the presence of cells earlier in the process of stepwise carcinogenesis face different challenges. There is evidence that some partially transformed cells progress very slowly (over decades) towards a clinically aggressive phenotype. Furthermore, carcinogenesis occurs in a parallel manner among at-risk cell lineages. In many organs, early neoplastic lesions are much morecommon than aggressive cancers. For example, polyps are morefrequent than cancers in the colon ${ }^{7}$, and there is evidence that approximately $40 \%$ of all men over the age of 60 years have prostate neoplasia that is detectable at autopsy, but only $3 \%$ have lesions that will affect their lifespan ${ }^{8,9}$.

PSA measurement is an important example of a sensitive screening method that can detect the presence of neoplastic cells relatively early in the transformation process, compared with most imagingbased screening techniques ${ }^{10}$. The rate of neoplastic progression in prostate cancer, as in other cancers, varies considerably between tumours. There is evidence that progression of a large subset of prostate neoplastic lesions towards an aggressive phenotype is slow, requiring years or even decades. If PSA or more sensitive screening methods were to allow identification of all men with some form of neoplasia, a situation would arise in which a large proportion of the elderly male population would 


\section{Timeline | Breast self-examination - changing attitudes}

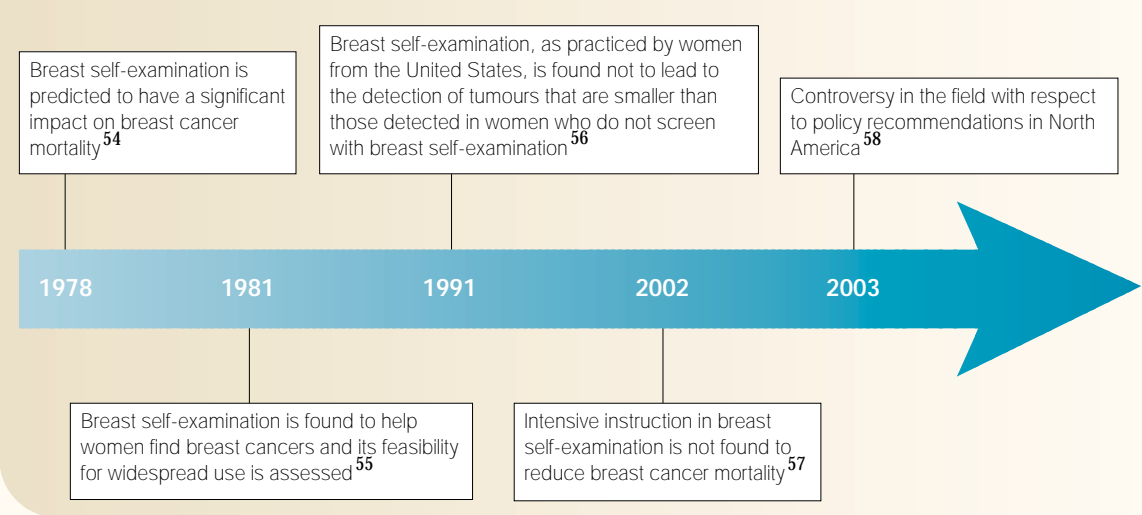

require intervention. Such screening results would not represent falsepositives in a technical sense, as neoplasia would, in fact, be present, but the results would be problematic clinically as the natural history of many (but not all) screening-detected lesions would befavourable.

In clinical practice, PSA screening often leads to difficult choices for men who discover the presence of lesions of uncertain prognosis. Clinicians who advisemen found to have elevated PSA on screening must first rule out frank false positives (related to prostatitis, for example), usually by biopsy. If neoplasia is confirmed, clinical judgment taking into account the patient's age, co-morbid conditions and clues regarding prognosis, such as the Gleason score - is needed to decide if intervention is in the patient's best interest. $M$ any patients, understanding that their lesion might or might not result in clinical illness if left untreated, choose to accept treatments with non-trivial morbidity 'just to be sure'. However, this might not provide the anticipated piece of mind, as many treated men experience anxiety concerning risk of recurrence ${ }^{11,12}$. Although there is a risk that PSA screening might fail to be of value because of highly aggressive interval cancers that can arise between sequential PSA measurements, in practice this is less frequently encountered than the detection of neoplastic lesions that are unlikely to result in clinical illness if left untreated, represented by tumour 3 in FIG. 1.

In contrast to certain screening methods for other cancers in which insufficient sensitivity is a significant limitation, PSA screening represents an example of a screening method in which the main limitation is that only subsets of those identified on screening requireintervention. The overall benefit of PSA screening programmes must take into account both the potentially life saving benefits of curative early intervention in men with aggressive prostate cancer, and the cost and morbidity of identifying and treating lesions that are not destined to cause significant clinical illness. The discovery of genetic, serum or tumour markers that could be used in conjunction with PSA to allow screening for the presence of the subset of lesions that are likely to lead to clinically important illness could rapidly alter thelandscape of prostatecancer screening. Research in this area is ongoing: recent data raises the possibility that serum analytes that are related to insulin growth factor (IGF) physiology might be related specifically to the risk of aggressive prostatecancer ${ }^{13,14}$.

This research topic is challenging because there is likely to be a strong stochastic component to the fate of individual partially transformed cells - some might accumulate further mutations that will result in malignant progression, whereas others might remain stable or even accumulatemutations that result in apoptotic death. A concept that deserves study is the notion that germline genetic factors - distinct from somatic-cell genetics - might influencethe speed of stepwise carcinogenesis, and so offer clues to identifying individuals in whom early lesions are particularly dangerous. D efective DNA repair represents one obvious example in this regard, but moresubtleinfluences involving the effects of hormonal milieu or the probability of survival as compared with apoptosis of at-risk cellsal so deserve investigation.

These challenges are more complex than conventional concerns of 'false-positive' and 'false-negative' screening tests. Conventional evaluations focus on the ability of a screening test to detect a lesion, with less emphasis on the definition of biological characteristics of lesions that are worth detecting. All would agree that identifying a cyst as a cancer is a false positive (and one that will be relatively easy to remedy with improved technology), but in a biological sense identifying and targeting for surgery an early cancer that is not destined to ever cause illness is also a false positive.

H eterogeneity. H eterogeneity between cells of a singlemacroscopic cancer is well documented. N ot only is there heterogeneity in the sense of different cell types (such as stromal, vascular and neoplastic) that comprise a tumour, but, more importantly, heterogeneity in gene expression is usually considerable even in analyses that are confined to the frankly neoplastic cells of a lesion ${ }^{15}$. A common example for clinicians is the heterogeneity of oestrogen-receptor expression among the individual malignant breast epithelial cells that comprise a breast cancer.

This heterogeneity complicates the development of tumour markers because the very notion of expression of a 'marker' by a 'tumour' is a simplification of the biological reality that, during the natural history of a particular neoplasm, the percentage of cells that express a given antigen will vary. N eoplastic progression towards aggressive cancer occurs simultaneously among many clonal populations, which independently undergo somatic-cell mutational events.

Sophisticated proteomic analysis of neoplastic cells obtained from a tumour specimen by laser capture microdissection might minimize concerns related to heterogeneity between neoplastic and stromal cells, but it does not address the problem of heterogeneity within the neoplastic cell population. Data obtained from a singlecancer represent an integration of expression information from many individual cells. Gene-expression signatures indicating aggressive neoplastic behaviour are being devel oped, but, if the proportion of cells with the signature is small, the signal might be obscured by a large majority of sample cells that do not express the signature. So, negative results for a serum marker that is used for screening might indicate an absence of cancer, but also the presence of a cancer at a stage where only a small percentage of the cancer cells express the marker. A special case that might show promise is the use of marker proteins that are necessary for an aggressive phenotype, rather than those that are simply associated with malignancy.

Small lesionscan beaggressive. Thenotion that cancer deaths in a population can be significantly reduced by public-health programmes based on the credo 'find it early and cut it out' 


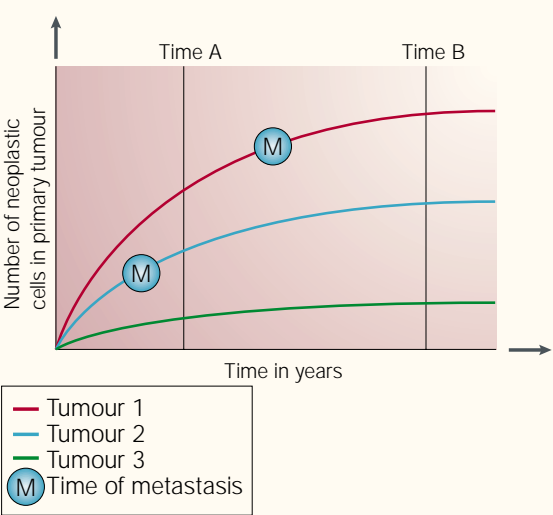

Figure 1 | When in the natural history of a cancer will screening be useful? This varies according to the biological behaviour of the tumour. In the illustration, ' $\mathrm{M}$ ' indicates the timepoint at which aggressive tumour cells metastasize. In the case of tumour 1 , screening and resection at time $A$ will result in cure of an otherwise lethal cancer whereas screening and resection at time $B$ will not prevent death from metastasis. In the case of tumour 2, screening at time A will represent a technical triumph, as the primary lesion is small and perhaps hard to detect, but will not be helpful, as this cancer has already metastasized. Detection at time $B$ will be of even less value. In the case of tumour 3 , the natural history is non-aggressive: successful screening at either time point will probably lead to interventions, possibly with associated risks, for a cancer that would never have caused a clinically important illness.

is simplistic, because the size of the primary lesion isonly imperfectly related to metastatic potential. Small-cell lung cancer (SCLC) provides an examplein which the probability of metastatic dissemination of even very small neoplasms is high. Small breast cancers areless likely than SCLC to metastasizeearly; however, the probability of metastatic spread before detection of breast cancers by screening is high enough that large numbers of women are offered adjuvant hormonal or chemotherapies following resection of apparently localized lesions. Thefact that such adjuvant therapies improvesurvival representsmedical progress, but, conversely, indicates that current screening does not always detect breast cancer early enough. Also problematic is the fact that, at present, no screening method, even accompanied by detailed ancillary investigations of screening-detected lesions, identifies with certainty those breast cancers in which surgical curecan beguaranteed, rendering adjuvant therapy unnecessary. Consequently, many women who are, in fact, surgically cured must undergo adjuvant therapies. Conversely, chemotherapy might not be offered to women with very small breast cancers. Thesetumours usually have a very good prognosis, but, in somecircumstances, such as in the presence of BRCA 1 mutations, small size and negative nodal statusmight not guaranteea good prognosis ${ }^{16}$. Identifying women who areBRCA1mutation carriers before diagnosis (or at least before treatment) might result in different therapeutic decisions. Recent progress ${ }^{17}$ in defining breast cancer gene-expression signatures that are associated with poor prognosis even in the presence of small tumour sizerepresents an important step towards the successful selection of patients for adjuvant treatment. However, thisemphasizes one of the challenges to effectivescreening: certain tumours might be life-threatening even if they are found beforethey becomelarge.

Varied risk, customized screening? Cancer screening methods might be applied universally according to age and gender, to selected subpopulations or to individuals. Thereare well-known groups (such as those with genetic predisposition or accidental carcinogen exposure) in which it might makesense to consider screening strategiesthat are different from those applied in the general population. H owever, the fact that a subgroup has a higher risk of cancer does not necessarily mean that moreintensivescreening will result in clinical benefits. Benefits will depend on the ability of the screening method to detect cancers at a curablestage.

This problem is exemplified by women who carry germline BRCA1 or BRCA2 mutations. Such women have a particularly high risk of developing breast cancer. The average age of onset of BRCA 1/2-related breast cancer is also at least 15 years younger than in the general population, so if mammography is going to influencesurvival in these women, it must be started no later than the fourth, or early in the fifth, decade of life. It might be expected that the positive predictive value of mammography would behigher for women at increased risk than for women at average risk. This might be offset, however, by their younger age of onset - when the breasts are expected to be denser and mammography is less effective at detecting neoplasia - and the generally unfavourablebiological characteristics of BRCA1/2-related breast cancers ${ }^{18}$. The typical BRCA1 tumour - a high-grade, oestrogen-receptor-negative, p53-positive cancer occurring in a young woman - has precisely the characteristics of those tumours that are most likely to be diagnosed in the interval between screening examinations ${ }^{19}$, and recent data show that BRCA1 tumours often present as 'interval cancers' 20 and are aggressive ${ }^{16}$. Interestingly, in the positive Swedish two-counties mammography trial, there was no survival benefit for women in the screened arm who developed high-grade invasi ve ductal carcinomas at 50 years of age or younger ${ }^{21}$. Furthermore, in a retrospective cohort of women who had been diagnosed with breast cancer and had been tested for BRCA1/2 mutations, mammography performed before surgery was significantly less likely to show a cancer if the woman was a mutation carrier and was either under 50 years of age at diagnosis, had a small tumour, or both ${ }^{22}$. So even if frequent screening were to be performed, BRCA1/2-related breast tumours might bemissed by mammography. These findings, when taken together, indicate that accepted techniques, such as mammography, might not significantly reduce breast cancer mortality in BRCA1/2-mutation carriers. Other imaging modalities, such as magnetic resonanceimaging ( $\mathrm{MI}$ ), will probably outperform mammography in BRCA1/2 carriers ${ }^{23,24}$, but the question of the appropriate screening interval for BRCA1/2-mutation carriers remains unanswered, and $M \mathrm{RI}$ studies in BRCA1/2-mutation carriers have yet to report medium-term follow-up data. Even if these studies show thebenefits of M RI, it might not be practical to shorten screening intervals to the extent that is necessary to detect curable cancers - four breast M RIs per year, even if possible, might not bea practical method for breast cancer control in high-risk women. Surgical prevention would probably bemore effective than screening ${ }^{25}$, but it has not been universally accepted by women at risk ${ }^{26}$. Increasing acceptance of surgical measures might lessen the need for effectivescreening for BRCA 1/2-related breast cancer, but it is difficult to imaginethat successful surgical intervention will completely obviate the need for better screening tests.

If it is assumed that the general population contains subpopulations that vary in the degree of benefit that screening would provide, the question of reviewing all individuals to identify the subpopulations for which screening should either be emphasized or avoided arises. 'Targeted' screening of selected subpopulations is appealing because the benefits of screening would increase if those who do not benefit - either because they are predisposed to cancers that are so aggressive that screening is unlikely to result in cures, or because they have a very low risk of cancer - were excluded. So far, few studies have addressed these issues, but interest is increasing. In breast cancer, for example, should the recommendations for women who are found to have mammographically dense breasts on initial screening be the same as for the general population? These 


\section{Box1 | Colorectal cancer screening: financial implications}

\author{
Annual cost estimate for universal colonoscopy at age 50 \\ - Number of individuals in the United States who will turn 50 during a 12-month period: \\ $3,000,000$ (REF. 52) \\ - Cost of screening colonoscopy per person: US\$ 1,000 (REF. 58) \\ -Annual cost of universal population-based screening by colonoscopy for 50 -year olds, \\ excluding costs related to follow-up of abnormalities detected: US $\$ 3,000,000,000$ \\ - Cost estimate in terms of dollars needed to save 1 year of life: US $\$ 10,000-25,000$ per year of \\ life saved ${ }^{53}$
}

women have an increased risk of breast cancer ${ }^{27}$, which might imply the potential for increased benefit. Conversely, mammography might be less able to accurately identify small lesions in these women ${ }^{24}$. Colorectal cancer provides another examplein which we need moreinformation to 'customize' screening recommen dations on the basis of known risk factors of modest magnitude, such as polymorphisms in known tumour-suppressor genes ${ }^{28}$ or high IGF1 levels ${ }^{29}$. In the more extreme case of certain hereditary polyposis syndromes, the role for colorectal screening is limited because the risks are so high that most favour colectomy before adulthood ${ }^{30}$

Lack of sensitive serum markers. Theidentification of reliable serum markers for detecting curable cancers has been a longstanding research goal ${ }^{31}$. Some markers, such as carcinoembryonic antigen (CEA), have been found to be useful aids for physicians in determining the response to treatment, but lack the sensitivity and specificity that is required for screening. PSA is more useful for screening than CEA, but its optimum use still remains controversial. Ongoing research using genomic approaches to analyse gene expression in tumours, or using proteomic approaches in the analysis of serum, might identify more sensitive markers than those that are available at present. However, this might create dilemmas. A positive 'cancer test' in the absence of symptoms or a lesion that can be detected by imaging would present difficult decisions for patients and physicians.

Financial obstacles to screening Colorectal cancer illustrates some of the serious financial implications of adopting a nationwidescrening programme. Although it is uncertain which methods are most effective, screening for colorectal cancer is generally regarded as useful in reducing colon cancer incidence and mortality ${ }^{32-34}$. But how much will it cost?
The data indicating that screening for colorectal cancer by sigmoidoscopy can savelives are convincing, but flexiblesigmoidoscopy as a first-linescreening tool has not gained acceptance. This is probably because a one-time flexiblesigmoidoscopy after a positive faecal occult blood test is thought to miss a quarter of all advanced cancers; these are beyond the reach of thesigmoidoscopeand do not havea distal polyp in association with the proximal cancer ${ }^{35}$. Randomized studies of one-time colonoscopy are in progress, and data available at present strongly indicate that colonoscopy every 10 years or even once at age 50 years would result in a reduction in colorectal cancer mortality ${ }^{32}$. Other, non-invasive techniques, such as 'virtual colonoscopy', might replace endoscopic techniques, but they remain underevaluated at present ${ }^{36}$.

Even colonoscopy is far from perfect. Recent research has questioned the universality of a simple adenoma-carcinoma progression model of colorectal carcinoma. In particular, studies of hyperplastic polyps have indicated that pathways to colorectal cancer might be more varied than was previously thought ${ }^{37}$. These findings could limit the effectiveness of screening and polypectomy as a method of reducing colorectal cancer mortality in individuals in whom stepwise carcinogenesis proceeds quickly.

Although colonoscopy still seems to bethe best alternative, the financial costs would be enormous (BOX 1). In fact, theimplementation of colonoscopy isnot even practical at present, as therearenot enough physicians performing colonoscopies. If the costs of colonoscopy could besubstantially reduced, this method might become a viable option ${ }^{34}$, but, at the moment, most societies will not be able to afford universal colonoscopy screening. Onceonly population-based flexiblesigmoidoscopy might bea practical alternative, even though it is acknowledged that it is not an ideal test. Cost-effectiveness analyses provide support for yearly re-hydrated faecal occult blood tests, combined with a sigmoidoscopy every 5 years from age 50 years to 85 years (REF. 33). A large multicentre randomized trail of once-only flexible sigmoidoscopy at age 60 years is now underway in the UK and Italy. The first analysis of mortality data can be expected in 2004 (REF. 38). If this trial shows important benefits, singleflexiblesigmoidoscopy by nurse practitioners will deserve consideration as a practical choice for population-based colorectal cancer screening, particularly in view of theevidence that nurse practitioners are capable of performing sigmoidoscopy to the same performance level as gastroenterologists $\mathrm{s}^{39}$. Of course, those who can pay for the 'best' screening test might choose more aggressiveor comprehensivescreening options, but this ishardly a way to determinethe best approach to populationbased screening, which, by its very nature, must befunded from taxation.

Elementary anatomical issues are relevant to the practicality of screening: one reason for the success of Pap screening for cancer of the uterine cervix is that screen-detected lesions can often be dealt with conveniently in a practitioner's office without the need for expensive or dangerous interventions. A finding that lung cancer mortality could be reduced by repeated spiral CT scans of at-risk populations would be problematic, particularly if the effect on mortality were small, not only because of the cost of implementing a screening programme, but also because of the need to increase bronchoscopy and/or thoracic-surgery capacity to deal with the screen-detected lesions, many of which will befalsepositives 4 .

\section{How to evaluate screening?}

Evaluation of the benefits of a screening method is complex. The first data that become available during formal or informal attempts to assess the value of a screening modality relate to the number of occult cancers detected. Detection of occult cancers is necessary but not sufficient for a screening method to be useful. An obvious hazard, particularly in efforts to screen for neoplasms such as small-cell lung cancers that tend to metastasize early, is that screening will identify occult cancers, but subsequent to metastasis. Even an impressive detection rate, in the absence of documentation of improved survival, cannot be used to justify theintroduction of a screening method into clinical practice. The current controversy regarding the value of mammography provides a clear example of difficulties that are related to statistical techniques used to evaluatescreening methodology 2,40.

In most cancer screening trials, disease-specific mortality is measured. In clinical trials of treatment, purists prefer to measure overall 
Box 2 | Evaluation of screening techniques

The argument for 'all-cause' mortality versus 'disease-specific' mortality ${ }^{41}$ : - Screening trials often measure 'disease-specific' mortality as the key outcome measure. This approach might be inadequate to fully reflect the impact of a screening test.

- D eaths caused, both directly and indirectly, by screening tests themselves might be incorrectly attributed to other causes. These deaths caused are often not accounted for as disease-related mortality.

- Deaths from cancer in the control group might be misattributed to other causes, or deaths in the screened group due to other causes are attributed to the screened cancer and are not accounted for as 'all cause'. This results in a'sticky diagnosis' because the cancer diagnosis 'attracts' other diseases that are, in fact, the true cause of death.

-This indicates that disease-specific mortality might be a suboptimal end point for screening trials and that a reduction in disease-specific mortality cannot be cited as strong evidence of efficacy when the all-cause mortality is the same or higher in the screened group.

-When all-cause mortality is considered to be an end point, it might be increasingly difficult to identify the benefits of screening for late-onset cancers such as ovarian, prostate and colorectal cancer, especially in an ageing population.

- Recent data on the use of hormone-replacement therapy as a way of reducing mortality rates in post-menopausal women, and the use of tamoxifen to prevent breast cancer, haveillustrated the problems with using measures other than all-cause mortality in evaluating an intervention that is designed to lower cancer mortality.

mortality, particularly when potentially dangeroustreatmentsareinvolved. Recent data from Black and colleagues, summarized in BOX 2, indicatethat it is also preferableto consider allcause mortality as the measured end point in trials of screening. This opinion is based on the re-analysis of 12 published, randomized trials of cancer screening (including seven of mammography) in which seven of the studies had all-cause and disease-specific mortality rates that went in opposite directions, or differed significantly in magnitude ${ }^{41}$. Interestingly, the analysis of Black et al. confirms ${ }^{41}$ that the Edinburgh breast cancer screening trial ${ }^{42}$ had highly biased randomization, as theall-cause mortality in the control group was significantly greater than the breast cancer mortality in this group. This might relate, at least in part, to social class differences in thescreened and observation groups.

It is likely that all-cause mortality is a less biased end point than cause-specific mortality. Therefore, when assessing the outcome of screening trials, it is important to remember that thetotal number of deathsin each arm of the study is ultimately more relevant to the success or failure of screening programmes than thenumber of any specific cause of death. This approach has been criticized by statisticians, radiologists and epidemiologists $s^{40,43,44}$. The main argument put forward by these critics is that many of the differences between cause-specific and all-cause mortality that were noted by Black and colleagues could be dueto chance, as deaths attributed to the cancer for which screening has been initiated will inevitably represent only a small fraction of the total number of deaths in a screened population. The central point that Black and colleagues re iteratein their response is that it is inherent in screening programmes that few will behelped, somewill beharmed and most will be unaffected ${ }^{45}$. Intemperate attacks ${ }^{46}$ on those prepared to question the existing dogma ${ }^{2,47}$ indicate theentrenched nature of the views held, and indicate that these issues will not easily be resolved.

Unfortunately, the end points that provide the most rigorous answers are the most expensive and time-consuming to obtain, requiring at least years and possibly decades. The time and resources required are such that it is unrealistic to formally evaluate all proposed screening methods. Only those that show particular promise in preliminary studies and seem feasible for general application if they prove useful can be studied. These are difficult decisions, and it is a matter for debate whether, for example, trials of spiral CT imaging of smokers ${ }^{4}$ represent a wise use of resources ${ }^{47}$.

Further challenges arise in areas of rapidly evolving technology that are related to screening. If serum proteomics or novel imaging methods show promise, how should it be determined when the method is optimized to a degree that would justify a significant trial? Advocates for the introduction of new screening methods are generally correct when they predict significant technical advances over the next decadedoes this argue for waiting, rather than undertaking, a trial based on soon-to-be suboptimal instrumentation?
O verall, these caveats are not meant to give the impression that progress in the development of screening methods is impossible. On the contrary, we believe that refinement of screening methods now in use, though challenging, is necessary if population-based screening is to fill its potential as a method of cancer control. If current screening methods were an unqualified success, questions as to its effectiveness would not result in such scientific and public controversy ${ }^{48}$

\section{Way forward}

The public's view of screening is fairly straightforward: if a cancer is found early, then cure is more likely and therefore screening is a good thing. The first part of the clause is generally true, but the second part is less clear. $M$ any recent publications do not make encouraging reading ${ }^{2,3}$. Not only are there significant methodological problems with the interpretation of the results of trials, but also, as we have suggested, particular subgroups differ from the general population with respect to absolute cancer risk, mechanisms of carcinogenesis and the characteristics of their cancers. This means that clinical trials of screening protocolsmight bemoreinformative if they stratify participants by biological and other markers of pre-existing risk. Results obtained in population-based 'all-risks' studies might not be translatable to individuals in specific risk groups and viceversa. A new look at the scientific rationale of screening studies is justified, and a renewed focus on primary prevention is warranted.

Advances in areas such as medical physics and serum proteomics are necessary but not sufficient to allow for significant improvements in the contribution of screening to cancer control. Also needed are bi ological and epidemiological information that will guide the application of new screening modalities in ways that will optimize reduction in cancer morbidity and mortality, as well as minimizing the number of unnecessary surgical interventions. In addition, attention must be given to the relationship between cancer screening and cancer prevention. It is imprecise to regard screening as a prevention strategy. Screening results in early detection, whereas successful prevention results in fewer invasive cancers and/or delayed carcinogenesis. Conventional screening must be combined with a treatment modality to beworthwhile.

Conventional models regard cancer as the target; in this context, the goal of screening is detection of macroscopic (but 


\section{PERS PECTIVES}

small) cancers, and subsequently undertaking curative surgery. But there might be more potential for screening than the detection of cancers that are clinically occult. TABLE 1 contrasts the goals of screening populations for incident cancers, screening for risk of cancer and screening to identify individuals who stand to ben efit from particular risk-reduction strategies.

As pointed out by Michael Sporn, it might be useful to consider the process of carcinogenesis as an alternative target ${ }^{49}$. In this context, screening would be directed towards identifying those individuals who have a particular propensity towards rapid rates of carcinogenesis on the basis of unusual exposure to carcinogens, a highrisk hormonal profile, a specific genetic predisposition or lifestyle factors. Having identified, through screening, those individuals with a particular mechanism that predisposes them to carcinogenesis, appropriate prevention interventions tailored to the at-risk individual might be offered.

We are far from achieving this kind of intervention in oncology, but a useful precedent is provided by everyday practice in cardiology. Screening to reduce mortality from myocardial infarction is not based on assessing the coronary arteries in asymptomatic individuals. Rather, it is commonplace to screen populations for particular modifiable risk factors that predispose to coronary artery disease (such as hypertension) and then offer appropriate prevention strategies. So, the screening is primarily designed to identify those with risk factors for heart disease, and to guide preventative interventions, rather than to identify those who already have occlusion of coronary arteries.

We speculate that the notion of screening populations to identify those who have the most to gain from specific risk-reduction strategies (as distinct from screening to detect early cancers) might becomemore common. A recent report ${ }^{50}$ presents clinical criteria that identify individuals who benefit most from the use of tamoxifen as a breast cancer prevention strategy. It is possiblethat genetic or hormonal measurements could improve these predictions. In a sense, detection of BRCA 1 mutations represents a prototype of this kind of screning - a positive result indicates risk, rather than the presence of cancer, and hasimplications for prevention (in this case, prophylactic surgery) rather than for the treatment of cancers that are al ready present.

Notwithstanding theenormous challenges, efforts to use population screening to control cancer morbidity and mortality will continue aslong as our success in treating advanced cancer remains limited. Somescreening methods, such as Pap tests for cancer of the cervix, are clearly useful in reducing the number of individuals who cometo medical attention with late-stage disease, and in reducing death rates ${ }^{51}$. Other screening methods, even those regarded in theolder literatureas obviously worthwhile - breast self-examination, for example- are being re-examined in thelight of the notion that 'number of cancers detected' might not be the best end point. At present, the field is in flux: just as the usefulness of older screening techniques and the methods used to evaluate

\section{Table 1 | Prevention and screening: distinct but related Screening for cancer Screening for risk Find cancers that are Find people that have high surgically curable or low risk \\ Example Mammography \\ Determining presence of germline BRCA1/2 mutation \\ Screening for potential to benefit from a prevention strategy \\ Identify people whose risk level is modifiable by a particular pharmacological or lifestyle intervention \\ None shown so far; a representative hypothesis is that efficacy of SERMs in reducing post- menopausal breast cancer risk will be greatest in those women with high circulating oestradiol levels}

At present, most screening procedures in oncology involve efforts to find incident asymptomatic cancers. Obviously, to be of benefit, this kind of screening must be combined with therapies, usually surgical, to remove the primary tumour. 'Screening' for genetic predisposition factors or for circulating hormone profiles associated with risk represents an effort to describe heterogeneity of risk within a population, rather than to identify those individuals with occult cancers. Finally, the concept of screening a population to identify those individuals who stand to benefit most from specific prevention strategies is at the interface between cancer screening research and cancer prevention research and deserves further study. The goal here is to identify subpopulations for whom particular prevention interventions would be most likely to be useful. Such information could result in more targeted application of prevention strategies, much as cardiologists direct antihypertensives not to everyone, but specifically to the subpopulation that, identified by blood pressure determination, is most likely to benefit. SERMs, selective oestrogen-receptor modulators. their benefits are being questioned, basic research is providing new imaging methods and serum markers, some of which deserve clinical evaluation.

\section{Michael N. Pollak and William D. Foulkes are in the Programs of Cancer Prevention and $C$ ancer Genetics McGill University, Montreal, Quebec, Canada H 3T 1E2. Correspondence to M.P. e-mail: michael.pollak@mcgill.ca} doi:10.1038/nrc1042

1. Baxter, N. Preventive health care, 2001 update: should women be routinely taught breast self-examination to screen for breast cancer? CMAJ 164, 1837-1846 (2001).

2. Olsen, O. \& Gotzsche, P. C. Cochrane review on screening for breast cancer with mammography. Lancet 358, 1340-1342 (2001)

3. Woolf, S. H. The accuracy and effectiveness of routine population screening with mammography, prostatespecific antigen, and prenatal ultrasound: a review of published scientific evidence Int I. Technol Assess. Health Care 17, 275-304 (2001)

4. Mulshine, J. L. Opinion: Screening for lung cancer: in pursuit of pre-metastatic disease. Nature Rev. Cancer 3 pursuit of pre-m

5. Petricoin, E. F. et al. Use of proteomics patterns in serum to identify ovarian cancer. Lancet 359, 572-577 (2002)

6. Nicholson, P. W. \& Harland, S. J. Survival prospects after screen-detection of prostate cancer. BJ U Int. 90 686-693 (2002)

7. Neugut, A. I., J acobson, J. S. \& Rella, V. A. Prevalence and incidence of colorectal adenomas and cancer in asymptomatic patients. Gastrointest. Endosc. Clin. N . Am. 7, 387-399 (1997).

8. Neal, D. E. \& Donovan, J. L. Prostate cancer: to screen or not to screen. Lancet Oncol. 1, 17-24 (2000).

Whitmore, W. F. Localized prostate cancer: management and detection issues. Lancet 343 , 1263-1267 (1994).

10. Bok, R. A. \& Small, E. J . Bloodborne biomolecular markers in prostate cancer development and progression. Nature Rev. Cancer 2, 918-926 (2002).

11. Lofters, A., J uffs, H. G., Pond, G. R. \& Tannock, I. F. 'PSA-itis': knowledge of serum prostate specific antigen and other causes of anxiety in men with metastatic prostate cancer. J . Urol. 168, 2516-2520 (2002).

12. Tannock, I. F. Eradication of a disease: how we cured symptomless prostate cancer. Lancet 359, 1341-1342 (2002).

13. Pollak, M. Insulin-like growth factors and prostate cancer. Epidemiol. Rev. 23, 59-66 (2001).

14. Chan, J. M. et al. Insulin-like growth factor-I (IGF-I) and IGF binding protein-3 as predictors of advanced-stage prostate cancer) Nat Cancer Inst 94, 1099-1106 (2002)

15. Greller, L. D., Tobin, F. L. \& Poste, G. Tumor heterogeneity and progression: conceptual foundations for modeling. Invasion Metastasis 16, 177-208 (1996).

16. Foulkes, W. D. et al. Primary node negative breast cance in BRCA1 mutation carriers has a poor outcome. Ann. Oncol. 11, 307-313 (2000).

17. Van de Vijver, M. J. et al. A gene-expresssion signature as a predictor of survival in breast cancer. N. Engl. J. Med. 347, 1999-2009 (2002)

18. Chappuis, P. O., Nethercot, V. \& Foulkes, W. D. Clinicopathological characteristics of BRCA1- and BRCA2related breast cancer. Sem. Surg. Oncol. 18, 287-295 (2000).

19. Porter, P. L. et al. Breast tumor characteristics as predictors of mammographic detection: comparison of interval-and screen-detected cancers. J. Natl Cancer Instit. 91, 2002-2008 (1999).

20. Brekelmans, C. T. et al. Effectiveness of breast cance surveillance in BRCA1/2 gene mutation carriers and women with high familial risk. J. Clin. Oncol. 19, 924-930 (2001).

21. Tabar, L., Duffy, S. W., Vitak, B., Chen, H.-H. \& Prevost, T. $C$. The natural history of breast carcinoma: what have we learned from screening? Cancer 86, 449-462 (1999).

22. Goffin, J ., Chappuis, P. O., Wong, N. \& Foulkes, W. D. Re: Magnetic resonance imaging and mammography in women with a hereditary risk of breast cancer. J. Natl Cancer Inst. 93, 1754-1755 (2001) 
23. Stoutjesdijk, M. J . et al. Magnetic resonance imaging and mammography in women with a hereditary risk of breast cancer. J. Natl Cancer Inst. 93, 1095-1102 (2001).

24. Warner, E. et al. Comparison of breast magnetic resonance imaging, mammography, and ultrasound fo surveillance of women at high risk for hereditary breast cancer. J. Clin. Oncol. 19, 3524-3531 (2001).

25. Meijers-Heijboer, H. et al. B reast cancer after prophylactic bilateral mastectomy in women with a BRCA1 or BRCA2 mutation. N. Engl. J. Med. 345 , 159-164 (2001)

26. J ulian-Reynier, C. M. et al. Women's attitudes toward preventive strategies for hereditary breast or ovarian carcinoma differ from one country to another: differences among English, French, and Canadian women. Cancer 92, 959-968 (2001)

27. Boyd, N., Lockwood, G., Byng, J ., Tritchler, D. \& Yaffe, M. Mammographic densities and breast cancer risk. Cancer Epidemiol. Biomark. Prev. 7, 1133-1144 (1998)

28. Laken, S. J . et al. Familial colorectal cancer in Ashkenazim due to a hypermutable tract in APC. Nature Genet. 17, 79-83 (1997).

29. $M a, J$. et al. Prospective study of colorectal cancer risk in men and plasma levels of insulin-like growth factor (IGF)-I and IGF-binding protein-3. J . Natl Cancer Inst. 91 620-625 (1999).

30. Giardiello, F. M., Brensinger, J . D. \& Petersen, G. M AGA technical review on hereditary colorectal cancer AGA technical review on hereditary colorectal cancer
and genetic testing. Gastroenterology 121, 198-213 and gen
(2001).

31. Sidransky, D. Emerging molecular markers of cancer Nature Rev. Cancer 2, 210-219 (2002).

32. Anderson, W. F. et al. Colorectal cancer screening fo persons at average risk. J . Natl Cancer Inst. 94, 1126-1133 (2002).

33. Frazier, A. L., Colditz, G. A., Fuchs, C. A. \& Kuntz, K $M$. Cost-effectiveness of screening for colorectal cancer in the general population. J AMA $\mathbf{2 8 4}$ 1954-1961 (2000).

34. Budenholzer, B. Cost-effectiveness of colorectal cancer screening. I AMA 285, 407 (2001).

35. Lieberman, D. A. \& Weiss, D. G. One-time screening for colorectal cancer with combined fecal occult-blood testing and examination of the distal colon. N. Engl. J testing and examination of the

36. Pineau, B. C. et al. Validation of virtual colonoscopy in the detection of colorectal polyps and masses: rationale for proper study design. Int. J. Gastrointest. Cancer 30 133-140 (2001).

37. J ass, J . R., Whitehall, V. L., Young, J \& \& Leggett, B. A. Emerging concepts in colorectal neoplasia. Gastroenterology 123, 862-876 (2002).

38. Atkin, W. S. et al. Design of a multicentre randomised trial to evaluate flexible sigmoidoscopy in colorectal cancer screening. J. Med. Screen. 8, 137-144 (2001).

39. Schoenfeld, P. et al. Accuracy of polyp detection by gastroenterologists and nurse endoscopists during flexible sigmoidoscopy: a randomized trial. flexible sigmoidoscopy: a randomized trial.

40. Kopans, D. B. The most recent breast cancer screening controversy about whether mammographic screening benefits women at any age: nonsense and nonscience. Am. J. Roentgenol. 180, 21-26 (2003).

41. Black, W. C., Haggstrom, D. A. \& Welch, G. All-cause mortality in randomized trials of cancer screening. J . Natl Cancer Inst. 94, 167-173 (2002).

42. Alexander, F. E. et al. 14 years of follow-up from the Edinburgh randomized trial of breast-cancer screening. Lancet 353, 1903-1908 (1999).

43. Church, T. R., Ederer, F. \& Mandel, J. S. Re: All-cause mortality in randomized trials of cancer screening. J. Natl Cancer Inst. 94, 861 (2002)

44. Gail, M. H. \& Katki, H. A. Re: All-cause mortality in randomized trials of cancer screening. I. Natl Cance Inst 94, 862 (2002).

45. Black, W. C., Haggstrom, D. A. \& Welch, H. G. Re: Allcause mortality in randomized trials of cancer screening. J. Natl Cancer Inst. 94, 865-866 (2002).

46. Rogers, L. F. Screening mammography: target of opportunity for the media. Am. J. Roentgenol. 180, 1 (2003)

47. Mahadevia, P. J . et al. Lung cancer screening with helical computed tomography in older adult smokers: a decision and cost-effectiveness analysis. J AMA 289, 313-322 (2003).

48. Kolata, G. Breast cancer: mammography finds more tumors. Then the debate begins. New York Times $(9 \mathrm{Apr}$ tumors

49. Sporn, M. D. \& Suh, N. Chemoprevention: an essential approach to controlling cancer. Nature Rev. Cancer $\mathbf{2}$ 537-543 (2002)
50. Veronesi, $U$. et al. Italian randomized trial among women with hysterectomy: tamoxifen and hormone-dependen breast cancer in high-risk women. J . Natl Cancer Inst. 95, 160-165 (2003)

51. Franco, E. L., Duarte-Franco, E. \& Ferenczy, A. Cervical cancer: epidemiology, prevention and the role of HPV infection. CMAJ 164, 1017-1025 (2001).

52. US Bureau of Census. An Aging World: 2001 [online] (cited $24 \mathrm{Feb} 2003$ ),

<http://www.census.gov/prod/2001pubs/p95-01-1.pdf> (2001).

53. Pignone, M., Saha, S., Hoerger, T. \& Mandelblatt, J. Cost-effectiveness analyses of colorectal cancer screening: a systematic review for the US Preventive Services Task Force. Ann. Intern. Med. 137, 96-104 Services

54. Greenwald, P. et al. Estimated effect of breast selfexamination and routine physician examinations on breast cancer mortality. N. Engl. J . Med. 299, 271-273 (1978).

55. Huguley, C. M. \& Brown, R. L. The value of breast selfexamination. Cancer 47, 989-995 (1981).

56. Newcomb, P. A. et al. B reast self-examination in relation to the occurrence of advanced breast cancer. J . Natl Cancer Inst. 83, 260-265 (1991).

57. Thomas, D. B. et al. Randomized trial of breast examination in Shanghai: final results. J . Natl Cancer Inst. 94, 1445-1457 (2002)
58. Smith, R. A., Cokkinides, V. \& Eyre, H. J. American Cancer Society guidelines for the early detection of cancer, 2003. CA Cancer J. Clin. 53, 27-43 (2003)

Acknowledgements

The authors would like to thank R. Narasimhadevara for her contribution to this review. W.D.F. is a Principal Investigator of the Canadian Genetic Diseases Network. M.N.P. holds the Alexander Goldfarb Research Chair in Medical Oncology at McGill University.

(4) Online links

\section{DATABASES}

The following terms in this article are linked online to: Cancer.gov: http://www.cancer.gov/cancer_information/ breast cancer | colon cancer | ovarian cancer | prostate cancer small-cell lung cancer

LocusLink: http://www.ncbi.nih.gov/LocusLink/

BRCA1 | BRCA2 | p53 | PSA

FURTHER INFORMATION

McGill Program in Cancer Genetics:

www.mcgill.ca/cancergenetics

McG ill Program in Cancer Prevention

www.mcgill.ca/cancerprev

Access to this interactive links box is free online.

\title{
Improving the evaluation of new cancer treatments: challenges and opportunities
}

\author{
M ace L. Rothenberg, D avid P. Carbone and D avid H. Johnson
}

There are, at present, ten times more anticancer drugs being tested in clinical trials than there were 15 years ago. Many of the new classes of agents, however, are predicted to work in only small subpopulations of patients, target unconventional aspects of tumour development and interact with other agents in an unpredictable manner. How can clinical trials be re-designed to accommodate the new features of targeted anticancer drugs?

In the dynamic new era of clinical cancer research, it is estimated that there are more 350 new agents that are in clinical development at present for cancer or cancer-related indications ${ }^{1}$. This is more than ten times the number of agents we had 15 years ago. Yet, along with this unprecedented opportunity, a sense of uncertainty and frustration permeates the fields of cancer treatment research and drug development. The apparently straightforward task of designing clinical trials that can clearly establish the beneficial impact of a new agent on a patient with cancer has become increasingly difficult. The problem lies in the fact that many of the new classes of agents are predicted to work only in small subpopulations of patients, affect tumours in an unconventional fashion and interact with other classical and novel agents in ways that we cannot yet predict. H ow can we resolve this clinical paradox and take full advantage of the opportunities for real progress that lie before us?

\section{The price of success}

In the 1990s, therewas a surge in the development and approval of new drugs for oncological indications. Thirty-six new agents were approved for the treatment of cancer and cancer-related indications in that decade - more than in the preceding 40 years (FIG. 1 ; and see FDA approval statistics in onlinelinks box). Similarly, the number of claims approved by theFood and Drug Administration (FDA) for thetreatment of cancer indications during the 1990s exceeded the number granted during the preceding 40 years (FIG. 2; and see FDA approval statistics in online links box). Identification of exploitablemolecular targets, 